\title{
Investigation of convergent and divergent genetic influences underlying schizophrenia and alcohol use disorder
}

Emma C. Johnson ${ }^{1}$, Manav Kapoor ${ }^{2}$, Alexander S. Hatoum ${ }^{1}$, Hang Zhou ${ }^{3,4}$, Renato Polimanti ${ }^{3,4}$, Frank R. Wendt ${ }^{3,4}$, Raymond K. Walters ${ }^{5,6}$, Dongbing Lai ${ }^{7}$, Rachel L. Kember ${ }^{8,9}$, Sarah Hartz ${ }^{1}$, Jacquelyn L. Meyers ${ }^{10,11}$, Roseann E. Peterson ${ }^{12}$, Stephan Ripke ${ }^{5,6,13}$, Tim B. Bigdeli ${ }^{10}$, Ayman H. Fanous ${ }^{10}$, Carlos N. Pato ${ }^{10}$, Michele T. Pato ${ }^{10}$, Alison M. Goate ${ }^{2}$, Henry R. Kranzler 8,9 , Michael C. O'Donovan ${ }^{14}$, James T.R. Walters ${ }^{14}$, Joel Gelernter ${ }^{3,4,15,16}$, Howard J. Edenberg ${ }^{7,17}$, Arpana Agrawal ${ }^{1}$

${ }^{1}$ Department of Psychiatry, Washington University School of Medicine, Saint Louis, MO, USA ${ }^{2}$ Department of Neuroscience, Icahn School of Medicine at Mount Sinai, New York, NY, USA ${ }^{3}$ Department of Psychiatry, Division of Human Genetics, Yale University School of Medicine, New Haven, CT, USA

${ }^{4}$ Department of Psychiatry, Veterans Affairs Connecticut Healthcare System, West Haven, CT, USA ${ }^{5}$ Analytic and Translational Genetics Unit, Department of Medicine, Massachusetts General Hospital and Harvard Medical School, Boston, MA, USA

${ }^{6}$ Stanley Center for Psychiatric Research, Broad Institute of MIT and Harvard, Cambridge, MA, USA

${ }^{7}$ Department of Medical and Molecular Genetics, Indiana University School of Medicine, Indianapolis, IN, USA

${ }^{8}$ Department of Psychiatry, University of Pennsylvania Perelman School of Medicine, Philadelphia, PA, USA

${ }^{9}$ VISN 4 MIRECC, Crescenz VAMC, Philadelphia, PA, USA

${ }^{10}$ Department of Psychiatry and Behavioral Sciences, SUNY Downstate Health Sciences University, Brooklyn, NY, USA

${ }^{11}$ Henri Begleiter Neurodynamics Laboratory, SUNY Downstate Health Sciences University, Brooklyn, NY, USA

${ }^{12}$ Department of Psychiatry, Virginia Institute for Psychiatric and Behavioral Genetics, Virginia Commonwealth University, Richmond, VA, USA

${ }^{13}$ Department of Psychiatry and Psychotherapy, Charité - Universitätsmedizin Berlin, Campus Mitte, Berlin, Germany

${ }^{14} \mathrm{MRC}$ Centre for Neuropsychiatric Genetics and Genomics, Division of Psychological Medicine and Clinical Neurosciences, Cardiff University School of Medicine, Cardiff, UK

${ }^{15}$ Department of Genetics, Yale University School of Medicine, New Haven, CT, USA

${ }^{16}$ Department of Neuroscience, Yale University School of Medicine, New Haven, CT, USA

${ }^{17}$ Department of Biochemistry and Molecular Biology, Indiana University School of Medicine, Indianapolis, IN, USA

Abstract: 169 words

Manuscript: 3,531 words

Figures: 3

Corresponding author: Emma C Johnson

Washington University School of Medicine

Dept. of Psychiatry, 660 S. Euclid, CB 8134

Saint Louis, MO 63110, USA

emma.c.johnson@wustl.edu 
medRxiv preprint doi: https://doi.org/10.1101/2020.10.27.20220186; this version posted December 16, 2020. The copyright holder for this preprint (which was not certified by peer review) is the author/funder, who has granted medRxiv a license to display the preprint in perpetuity.

It is made available under a CC-BY-NC-ND 4.0 International license.

\begin{abstract}
Background: Alcohol use disorder (AUD) and schizophrenia (SCZ) frequently co-occur, and recent genome-wide association studies (GWAS) have identified significant genetic correlations between them.
\end{abstract}

Methods: We used the largest published GWAS for AUD (total cases $=77,822$ ) and SCZ (total cases $=46,827$ ) to systematically identify genetic variants that influence both disorders (in either the same or opposite direction of effect) as well as disorder-specific loci.

Results: We identified 55 independent genome-wide significant SNPs with the same direction of effect on AUD and SCZ, 8 with robust effects in opposite directions, and 98 with disorderspecific effects. We also found evidence for 12 genes whose pleiotropic associations with AUD and SCZ are consistent with mediation via gene expression in the prefrontal cortex. The genetic covariance between AUD and SCZ was concentrated in genomic regions functional in brain tissues $(p=0.001)$.

Conclusions: Our findings provide further evidence that SCZ shares meaningful genetic overlap with AUD, and suggest that genetic variants with an effect on both disorders are expressed in brain tissues. 


\section{Introduction}

Schizophrenia (SCZ) and alcohol use disorder (AUD) are serious psychiatric disorders ${ }^{1}$. AUD is more common in individuals with SCZ (prevalence of $20-30 \%{ }^{2,3}$, compared to $\sim 6 \%$ in the general population ${ }^{4}$ ) and a diagnosis of both is associated with greater psychiatric comorbidity ${ }^{5}$, more clinical complications ${ }^{6}$, and a lower likelihood of sustained medication adherence ${ }^{7}$ than either disorder alone. Both SCZ and AUD are moderately to highly heritable (twin- $h^{2}$ for SCZ = $81 \%^{8}$, AUD $=49 \%^{9}$ ), and genome-wide association studies (GWAS) have consistently found positive genetic correlations of AUD with SCZ (e.g., $\left.r_{g}=0.34, p=3.7 e-21\right)^{10}$. Further, polygenic risk scores (PRS) for AUD are significantly associated with SCZ risk ${ }^{10}$ and vice versa ${ }^{11}$. In contrast, the genetic correlation between SCZ and measures of typical alcohol consumption is weak (e.g., drinks/week: $r_{g}=0.01, p=0.67^{12}$ ), suggesting that SCZ might share substantial genetic liability only with the psychopathological aspects of disordered drinking ${ }^{13}$ and not alcohol consumption per se.

Despite the substantial genetic correlation between AUD and SCZ, there is little known regarding the underlying pleiotropic mechanisms in terms of the specific risk alleles, genes, and molecular pathways involved. Although recent studies have begun to elucidate the contributions of pleiotropic loci to shared genetic variance amongst disorders and complex traits ${ }^{14,15}$, these efforts have not included AUD, one of the most common psychiatric disorders. In addition to loci with a similar direction of effect on both disorders, modern cross-disorder GWAS methods can also identify divergent variants, i.e., those that are pleiotropic for two disorders but whose effect alleles operate in opposite directions, conferring risk for one disorder and protective effects for the other (e.g., potassium ion response genes that distinguish SCZ from bipolar disorder ${ }^{16}$ ). The identification of such variants is fundamental to identifying the pathways that contribute to diagnostic boundaries.

The current study outlines the nature of the shared genetic underpinnings of AUD and SCZ by conducting cross-disorder analyses of large genome-wide datasets of both Europeanand African-ancestry individuals (see Figure 1 for overview). We conducted ancestry-specific cross-disorder meta-analyses to systematically identify pleiotropic loci with significant convergent and divergent effects on both SCZ and AUD, and loci specific to each disorder. We also linked pleiotropic variants to gene expression data from the frontal cortex in an effort to prioritize the genes that are more likely to be causal. Because the extent to which the correlation between AUD and SCZ is attributable to salient functional categories remains unknown, we partitioned the genetic covariance between AUD and SCZ into relevant annotations. 


\section{Methods and Materials}

\section{Samples}

Alcohol Use Disorder

For the European-ancestry subset, we utilized GWAS data from the largest available AUD meta-analysis ${ }^{17}\left(\mathrm{~N}=313,959 ; \mathrm{N}_{\text {cases }}=57,564\right)$. This study meta-analyzed AUD GWAS data from the Million Veteran Program (MVP), where case status was derived from International Classification of Diseases (ICD) codes of alcohol-related diagnoses in electronic health records (EHR) data, and the Psychiatric Genomics Consortium (PGC) alcohol dependence GWAS ${ }^{18}$ with cases based on DSM-IV diagnoses.

We meta-analyzed two published GWAS ourselves to create the African-ancestry subset: the MVP Phase 1 GWAS of $\operatorname{AUD}^{10}\left(\mathrm{~N}=56,648 ; N_{\text {cases }}=17,267\right)$, and the PGC GWAS ${ }^{18}$ $\left(\mathrm{N}=5,799 ; \mathrm{N}_{\text {cases }}=2,991\right)$. We used METAL ${ }^{19}$ (which combines genome-wide summary statistics across multiple samples) to generate the African-ancestry summary statistics by metaanalyzing the GWAS data from the MVP Phase 1 AUD and PGC alcohol dependence GWAS using an inverse variance-weighted fixed-effects model, excluding SNPs with INFO score $<0.8$ and/or minor allele frequency $<0.01$ within each sample.

\section{Schizophrenia}

For the European-ancestry sample, we used the PGC Phase 2 + CLOZUK (a sample of individuals with schizophrenia who were treated with clozapine) SCZ GWAS meta-analysis ${ }^{20}$ (total $N=105,318 ; N_{\text {cases }}=40,675$ ). We used the summary statistics from the Genomic Psychiatry Cohort (GPC) SCZ GWAS ${ }^{21}\left(N=10,070 ; N_{\text {cases }}=6,152\right)$ for the African-ancestry cross-disorder analysis.

\section{Analysis}

Cross-disorder association analysis

We used "Association analysis based on SubSETs" (ASSET) 22 to combine the genomewide association data for AUD and SCZ (separately by ancestry), using the two-tailed metaanalysis approach to obtain a single cross-disorder association statistic, correcting for sample overlap. Unlike traditional meta-analysis approaches, ASSET takes into account SNPs with significant effects on multiple disorders even if the effects on the traits are in opposite directions. Default parameters were applied using the "h.traits" function, and we used LD Score Regression ${ }^{23,24}$ (LDSC) to obtain a rough estimate of sample overlap, which we accounted for in the additional covariance term. We then separated the ASSET results into four subsets: a 
"convergent" subset (effect allele with the same direction of effect for both disorders), a "divergent" subset (effect allele with opposite directions of effect on the disorders), a subset of SNPs with AUD-specific effects, and a subset of SNPs with effects only on SCZ (Supplemental Figure 1).

In the European-ancestry sample, we then uploaded the subset results (i.e., convergent, divergent, AUD-only, and SCZ-only SNPs from ASSET output) to FUMA v1.3.6a ${ }^{25}$ for annotation and identification of genome-wide significant risk loci and independent lead SNPs. We further subset these convergent and divergent loci to exclude top lead SNPs with $p>0.05$ in either individual disorder GWAS, to create a more conservative set of cross-disorder variants with at least nominal significance in both disorders (although the full subsets were used for gene-set and pathway analyses).

In the African-ancestry samples, which were smaller and accordingly lacked power for more extensive analyses, we focused on the overall set of pleiotropic cross-disorder variants, rather than parsing the pleiotropic variants into subsets with convergent and divergent effects. In addition, as much of the data required for follow-up analyses described below were restricted to European-ancestry individuals, these follow-up analyses were not conducted on the Africanancestry cross-disorder summary statistics.

Gene, gene-set, and pathway analyses

In the European-ancestry samples, gene-based analyses in MAGMA (v1.08) ${ }^{26}$ were conducted on the subset results (i.e., convergent and divergent SNPs from ASSET output) via the FUMA ${ }^{25}$ platform. These analyses included gene-set analyses using curated gene sets and GO terms from MsigDB ${ }^{27}$ (an online collection of annotated gene-sets), gene-property analyses based on tissue expression data from GTEx ${ }^{28}$ v8 (a repository of expression data by brain region from autopsies of 960 donors), and data visualization (more details in Supplemental Materials).

\section{SMR eQTL analyses and differential gene expression analyses}

To examine whether the effects of pleiotropic variants with convergent effects on AUD and SCZ may be mediated by gene expression patterns, we conducted a summary data-based Mendelian randomization ${ }^{29}$ (SMR) analysis on a set of expression quantitative trait loci (eQTL) data in the prefrontal cortex (meta-analyzed to combine data from ROSMAP ${ }^{30}$, PsychENCODE ${ }^{31}$, and COGA-INIA datasets ${ }^{32}$; total $\left.N=1,986\right)$. SMR is a Mendelian randomization-based analysis that integrates GWAS summary statistics with eQTL data to test 
whether the effect size of a SNP on the phenotype of interest is mediated by gene expression. SMR does not require raw eQTL data to build the weights. We excluded variants with pleiotropic effects significantly different from what would be expected under a causal model using the HEIDI-outlier method ${ }^{33}$ (excluding SNPs with HEIDI-outlier $p<0.05$ ).

We also examined whether genes mapped to pleiotropic loci by MAGMA (with $p<0.05$ ) were significantly enriched for genes showing differential expression $(p<0.05)$ in the prefrontal cortex using two comparisons in independent samples: we compared gene expression in 65 individuals with alcohol dependence and 73 healthy controls ${ }^{32}$, and 258 individuals with SCZ and 279 controls $^{34}$ (details in Supplemental Note) using Fisher's exact test ${ }^{35}$.

\section{Genetic correlations and partitioned covariance}

We used LDSC ${ }^{23,24}$ to estimate the genetic correlations $\left(r_{g}\right)$ between AUD, SCZ,-and two negative control traits (height and chronic ischemic heart disease). We compared the genetic correlations between AUD and SCZ, and DPW and SCZ using a block jackknife method implemented through LDSC to test the null hypothesis that $r_{g}(A U D, S C Z)$ minus $r_{g}(D P W, S C Z)=$ 0 .

We also used LDSC applied to Specifically Expressed Genes (LDSC-SEG ${ }^{36}$ ) to estimate the enrichment of AUD and SCZ across 13 specific brain regions (annotations defined using GTEx ${ }^{37}$ gene expression data).

We used GeNetic cOVariance Analyzer (GNOVA) ${ }^{38}$ to partition the genetic covariance $\left(\rho_{g}\right)$ between AUD and SCZ into salient annotation categories. These included 1) functional vs. non-functional areas of the genome (GenoCanyon ${ }^{39}$ annotations, defined by integrating genomic conservation measures and biochemical annotation data to generate a functional potential score for each genetic variant), 2) tissue- and regional-specific functionality (GenoSkyline ${ }^{40,41}$ annotations, which are tissue-specific functional regions defined by integrating high-throughput epigenetic annotations, 3) GTEx v6 ${ }^{37}$ brain region annotations from LDSC celltype specific analyses), and 4) minor allele frequency quartiles. We excluded the MHC region (chr6:26000885 - chr6:33999991) from both the LDSC-SEG and GNOVA analyses due to the long-range and complex LD in this region.

In sensitivity analyses conducted on individuals of European ancestry in the UK Biobank (from the Neale lab GWAS: https://www.nealelab.is/uk-biobank), we calculated the genetic correlation and partitioned genetic covariance between AUD, SCZ, and two negative control traits: height $(\mathrm{N}=360,388)$ and chronic ischemic heart disease $\left(\mathrm{CHD} ; \mathrm{N}=361,194 ; \mathrm{N}_{\text {cases }}=\right.$ 12,769). 


\section{Results}

Identifying pleiotropic variants, genes, and pathways in individuals of European ancestry

The cross-disorder analysis of AUD and SCZ in European-ancestry individuals identified numerous significant pleiotropic loci: after genome-wide clumping via FUMA ${ }^{25}$, there were 55 independent risk loci (with 60 lead SNPs) with convergent effects and 44 risk loci (56 lead SNPs) with divergent effects (i.e., effect allele with opposite effects on AUD and SCZ;

Supplemental Figure 1, Supplemental Tables 1-3). We also identified disorder-specific loci through ASSET: 90 with SCZ-only effects, and 8 with AUD-only effects (Supplemental Tables

4-5). MAGMA gene-based analyses identified 119 significant genes from the convergent subset and 105 genes from the divergent subset (Supplemental Figure 2, Supplemental Tables 6-7).

As ASSET ${ }^{22}$ searches for and determines the most likely subset for each SNP (i.e., classifying SNPs as having an effect only on AUD, only on SCZ, or on both disorders), some of the pleiotropic SNPs identified by ASSET were only significant in one of the single-disorder GWAS. For a more conservative description of pleiotropic loci of divergent effect, we further considered only the 8 loci where the top lead SNPs had opposite effects but $p<0.05$ for both AUD and SCZ (Supplemental Table 3; the convergent lead SNPs already had $p<0.05$ for both disorders). In the convergent subset, the strongest association was on chromosome 11 (lead SNP rs6589386, cross-disorder $p=5.7 e-18$; AUD $p=7.1 e-12$, SCZ $p=1.6 e-8)$. The strongest divergent signal was on chromosome 4 (lead SNP rs13135092, cross-disorder $p=2.9 e-31$; AUD $p=4.9 \mathrm{e}-18$, SCZ $\mathrm{p}=7.9 \mathrm{e}-16)$, located in an intron of SLC39A8. Importantly, although rs13135092 is pleiotropic for both AUD and SCZ, the same effect allele (A) increases risk for AUD and decreases risk (i.e., is protective) for SCZ.

MAGMA competitive gene-set analyses identified 2 significant GO terms in the convergent subset of variants, one related to DNA binding (GO: 0043565, $p=1.3 e-6$ ) and one related to neuronal differentiation (GO: 0045664, $p=1.9 \mathrm{e}-6$; Supplemental Table 8). There were no significant gene-sets identified for the divergent subset).

Both the convergent and divergent subsets of variants showed enrichment in all 13 brain tissues in MAGMA gene-property analyses, and the divergent subset also showed enrichment in pituitary tissues. (Supplemental Figure 3).

\section{Cross-disorder variants in African ancestry individuals}

In the African-ancestry cross-disorder analysis, there was limited power to identify pleiotropic loci, with results appearing to be primarily driven by the larger AUD GWAS (e.g., the 
one genome-wide significant SNP that ASSET identified as being pleiotropic had $p>0.05$ for SCZ).

Integration of eQTL data and differential gene expression in EUR-ancestry samples

Of the 22 genes that survived Bonferroni correction (for the number of genes tested; PSMR < 1.16e-5), SMR analyses identified 12 convergent genes whose cross-disorder associations with AUD and SCZ were consistent with mediation via gene expression in the prefrontal cortex (PFC; the remaining 10 genes with $\mathrm{p}_{S M R}<1.16 \mathrm{e}-5$ had $\mathrm{p}_{\mathrm{HEIDI}}<0.05$, indicating pleiotropy outlier status; Figure 2; significant results in Supplemental Table 10; full results in

\section{Supplemental Table 11).}

Neither the genes with variants of convergent nor divergent effect were enriched in specific differential gene expression analyses of postmortem PFC tissue of individuals with SCZ $\left(\mathrm{N}_{\text {cases }}=258\right)$ vs. controls or of AUD $\left(\mathrm{N}_{\text {cases }}=65\right)$ vs. controls.

\section{Genetic covariance and correlation}

There were significant positive genetic correlations $\left(r_{g}\right)$ between AUD and SCZ $\left(r_{g}=\right.$ $0.392, p=1.2 \mathrm{e}-42)$. In sensitivity tests, the negative control measure of chronic ischemic heart disease was not significantly correlated with AUD but height showed a small negative correlation $\left(r_{g}=-0.093, S E=0.021, p=7.54 e-6\right)$. SCZ showed a nominal genetic correlation with heart disease $\left(r_{g}=-0.063\right.$, SE $\left.=0.029, p=0.032\right)$, but none with height.

LDSC-SEG analyses that partitioned heritability enrichment by tissue-specific gene expression revealed significant enrichment for SCZ in three of 13 brain regions: the cortex, frontal cortex, and anterior cingulate cortex (FDR q-values < 2e-5), and for AUD in the anterior cingulate cortex (FDR q-value = 0.007; Supplemental Figure 4).

The genetic covariance $\left(\rho_{g}\right)$ of AUD and SCZ was significantly attributable both to functional regions of the genome $(p=1.0 \mathrm{e}-8)$, including those specifically functional in brain tissues ( $p=0.001$; see Figure 3a, Supplemental Table 12) and non-functional regions $(p=$ 1.5e-19; Supplemental Table 13), as well as all minor allele frequency quartiles except the lowest frequency quartile ( $p=2.9 e-5$ - 3.9e-9; Supplemental Table 14). While the point estimate of genetic covariance was highest in genes functional in immune tissues $\left(\rho_{g}=0.017\right)$, this estimate had a relatively large standard error (0.007) and did not reach significance after multiple testing corrections. There were no significant findings when partitioning the genetic covariance between AUD and height or SCZ and heart disease into tissue-specific categories with GNOVA. 
medRxiv preprint doi: https://doi.org/10.1101/2020.10.27.20220186; this version posted December 16, 2020. The copyright holder for this preprint (which was not certified by peer review) is the author/funder, who has granted medRxiv a license to display the preprint in perpetuity.

It is made available under a CC-BY-NC-ND 4.0 International license .

Because the genetic covariance of AUD and SCZ was significantly concentrated in brain tissues, we partitioned it further into specific brain regions, using the 13 GTEx brain region annotations provided by LDSC-SEG ${ }^{36}$. We found significant concentrations of genetic covariance in all 13 brain regions tested, with the greatest concentrations in the anterior cingulate cortex $(p=7.6 e-12)$, frontal cortex $(p=3.3 e-9)$, cortex $(p=1.2 e-7)$, and amygdala $(p=$ 3.1e-9; Supplemental Table 15, Figure 3b).

\section{Discussion}

The prevalence of AUD is elevated in those with SCZ, relative to the general population. Recent large GWAS of AUD document robust genetic correlations between AUD and SCZ ${ }^{17}$. Utilizing a subset-based meta-analytic approach, our cross-disorder analysis of AUD and SCZ identified loci with either the same or opposite direction of effects on AUD and SCZ; 55 convergent loci and 8 divergent loci had lead SNPs that were $p<0.05$ in both individual disorders. The genetic covariance between AUD and SCZ was concentrated in genes functional in the brain, and eQTL analyses of the convergent subset of variants (i.e., those with the same direction of effect on both disorders) identified 12 genes whose association with AUD and SCZ may be mediated via gene expression in brain tissues.

AUD and SCZ were robustly genetically correlated. In contrast, prior studies have found that SCZ is uncorrelated with measures of typical alcohol consumption (e.g., with drinks/week, $r_{g}$ $=0.01, p=0.67^{12}$ ), especially when alcohol frequency of use alone is considered ${ }^{42}$. Genetic correlations with indices that encompass heavy episodic drinking, such as the consumption subscale of the Alcohol Use Disorders Identification Test (AUDIT-C), are also lower $\left(r_{g}=-0.0003\right.$ $0.04)^{10,13}$ than those noted for AUD. A recent study has implicated that indices of socioeconomic status may influence genetic correlations between measures of substance use and psychopathology 43 ; however, even after co-varying for SES, the genetic correlation between alcohol frequency and SCZ remained non-significant. The genetic correlation between alcohol quantity and SCZ remained significant and relatively unchanged $\left(r_{g}=0.14-0.16\right)$, but markedly lower than the AUD-SCZ genetic correlation. This suggests that SCZ shares genetic variation primarily with the psychopathological aspects of problem drinking and AUD, even after accounting for socioeconomic measures.

The top divergent SNP, rs13135092, in an intron of SLC39A, showed strong associations with both AUD ( $p=4.9 \mathrm{e}-18)$ and SCZ $(\mathrm{p}=7.9 \mathrm{e}-16)$, with the A allele exerting a risk-increasing effect on AUD and a protective effect on SCZ. Comparison of summary statistics for over 4,756 traits ${ }^{44}$ indicated that the A allele was also associated with greater alcohol 
consumption, greater risk-taking, higher waist-hip ratio, lower bioelectrical impedance (i.e., greater adiposity) and higher systolic blood pressure, consistent with the direction of genetic association between these measures and AUD. However, the A allele was also associated with increasing cognitive performance, higher intelligence and with higher educational attainment while the direction of these effects is consistent with the "protective" effect of the A allele on risk for SCZ, it contradicts prior research showing an inverse genetic correlation between educational attainment and AUD. Given the convergent direction of associations with AUD and alcohol consumption, as well as aspects of risk-taking and cardio-metabolic traits, but divergent direction of associations with SCZ as well as cognition, we speculate that the A allele of this SNP may be related to milder AUD, typified by positive reward-related drinking and impulsivity that is effectively regulated by enhanced cognitive functioning.

Our top convergent SNP, rs6589386 is intergenic and an eQTL for DRD2 in cerebellar hemisphere tissue; this gene has been implicated for both AUD and SCZ across recent GWAS. In addition to AUD and SCZ, this variant has been implicated in GWAS of neuroticism, subjective well-being, alcohol consumption, and cigarette smoking.

Linking cross-disorder associations to eQTLs in the prefrontal cortex (PFC) suggested that the effects of pleiotropic variants on AUD and SCZ may be mediated by expression levels of several genes (Figure 2). Several of the identified genes were previously implicated in GWAS of metabolic traits (including NAT8, TRPS1 (up-regulated), BAG5, PPP1R13B (downregulated)), immunological traits (e.g., RERE, TRPS1, both up-regulated) and psychiatric phenotypes (e.g., up-regulated: $L R P 8$, down-regulated: PPP1R13B). Although these findings may not be limited to gene expression in the PFC, the limited data currently available do not permit examination of whether these findings extend to other brain regions.

While there are limited samples available with measured gene expression in post mortem brain tissue, particularly for substance use disorders, we used two independent datasets (alcohol dependent and control samples from New South Wales Tissue Resource Centre at the University of Sydney, schizophrenia and control samples from the CommonMind consortium) to link pleiotropic variants to differential gene expression. We found no evidence for enrichment of genes pleiotropically associated with both AUD and SCZ when considering genes differentially expressed in the brains of individuals with AUD or SCZ (compared to respective controls). However, our lack of findings could be due to limited statistical power in the RNAseq whole-genome transcriptome datasets $(N=138$ - 537$)$ relative to the GWAS datasets (Ns > $100,000)$. 
There are several limitations of the current study. First, nearly all gene expression reference datasets and other bioinformatic resources have been trained on European-ancestry samples. In addition, our African-ancestry samples were under-powered relative to the European-ancestry samples, and the SCZ sample more so than the AUD sample. Thus, the African-ancestry results were limited to the ASSET cross-disorder analysis, and appeared to be driven almost entirely by the larger AUD GWAS. Our motivation for conducting this crossdisorder analysis is because most cross-disorder GWAS rely on European-ancestry samples, generating inequity in downstream analyses of non-European populations. For instance, summary statistics from this cross-disorder GWAS could be used to generate polygenic risk scores in independent cohorts of African-American participants. Nonetheless, vast increases in African ancestry sample sizes are needed for studies of serious psychiatric conditions. Second, given the available data, we were limited to studying common genetic variation (minor allele frequencies $>1 \%$ ); thus, there may be rare variants of importance underlying the comorbidity between AUD and SCZ that are outside the scope of the current study. Third, in our sensitivity analysis, we found a significant negative genetic correlation between AUD and height $\left(r_{g}=-\right.$ $0.093, S E=0.021, p=7.54 e-6)$, which is likely driven in part by the very large sample sizes and polygenic nature of both traits, though there is some evidence for this relationship in the literature. One previous study found a negative genetic correlation between alcohol consumption and childhood height in males $^{45}\left(r_{g}=-0.23, p=0.002\right)$ and another study found a negative correlation between problematic alcohol use and comparative height at age $10\left(r_{g}=-\right.$ $0.09, p=7.3 e-5)$, though this was not significant when a Bonferroni correction was applied for the 715 traits tested ${ }^{17}$. Finally, while most of the AUD samples were screened for SCZ, some individuals among the SCZ samples very likely also had AUD. Unfortunately, there are no data currently available on which SCZ samples were screened for substance use disorders, to permit an estimation of such a bias.

Cross-disorder GWAS provide insights into genetic sources of comorbidity. While prior cross-disorder studies have provided foundational results for common psychiatric disorders, ours is amongst the first studies to focus on identifying pleiotropic loci for a substance use disorder, AUD, and SCZ. To understand the shared biology between substance use disorders and other psychiatric disorders, future efforts must include large numbers of individuals with AUD or problematic alcohol use rather than simple measures of consumption. 


\section{FUNDING}

ECJ was supported by grant F32AA027435. RP was supported by R21DA047527 and R21DC018098. FRW was supported by F32MH122058. AA is supported by K02DA032573. REP is supported by NIMH K01MH113848 and The Brain \& Behavior Research Foundation NARSAD grant 28632 P\&S Fund.

This work was conducted using the summary statistics from the Psychiatric Genomics Consortium's Substance Use Disorders Working group. The Psychiatric Genomics Consortium's Substance Use Disorders (PGC-SUD) working group is supported by MH109532 with funding from NIMH and NIDA.

The GPC was supported by grants R01 MH085548 and R01 MH104964 from the National Institute of Mental Health (NIMH), and genotyping of samples was provided by the Stanley Center for Psychiatric Research at Broad Institute.

COGS was supported by grants R01 MH065571, R01 MH065588, R01 MH065562, R01 MH065707, R01 MH065554, R01 MH065578, R01 MH065558, R01 MH86135, and R01 MH094320 from the National Institute of Mental Health.

Funding support for the Whole Genome Association Study of Bipolar Disorder and the GenomeWide Association of Schizophrenia Study was provided by the NIMH (R01 MH67257, R01 MH59588, R01 MH59571, R01 MH59565, R01 MH59587, R01 MH60870, R01 MH59566, R01 MH59586, R01 MH61675, R01 MH60879, R01 MH81800, U01 MH46276, U01 MH46289, U01 MH46318, U01 MH79469, and U01 MH79470) and the genotyping of samples was provided through the Genetic Association Information Network (GAIN).

This research is based, in part, on data from the Million Veteran Program, Office of Research and Development, Veterans Health Administration, with support from award \#I01 BX003341 and the VISN 4 Mental IIIness Research, Education and Clinical Center. This publication does not represent the views of the Department of Veterans Affairs or the United States Government.

\section{FINANCIAL DISCLOSURES}

Dr. Kranzler is a member of an advisory board for Dicerna Pharmaceuticals and a member of the American Society of Clinical Psychopharmacology's Alcohol Clinical Trials Initiative, which was supported in the last three years by AbbVie, Alkermes, Dicerna, Ethypharm, Indivior, Lilly, 
Lundbeck, Otsuka, Pfizer, Arbor, and Amygdala Neurosciences. Dr. Kranzler and Dr. Gelernter are named as inventors on PCT patent application \#15/878,640 entitled: "Genotype-guided dosing of opioid agonists," filed January 24, 2018. Drs. Johnson, Kapoor, Hatoum, Zhou, Polimanti, Wendt, R.K. Walters, Lai, Kember, Hartz, Meyers, Peterson, Ripke, Bigdeli, Fanous, C.N. Pato, M.T. Pato, Goate, O’Donovan, J.T.R. Walters, Edenberg, and Agrawal have no disclosures or conflicts of interest to report.

\section{ACKNOWLEDGEMENTS}

The Psychiatric Genomics Consortium's Substance Use Disorders (PGC-SUD) working group gratefully acknowledges prior support from NIAAA and thanks all our contributing investigators and study participants who make this research possible. We acknowledge collaborations with the Million Veteran Program, Psychiatric Genomics Consortium Substance Use Disorders Working Group, and Genomic Psychiatry Cohort Investigators. A list of Genomic Psychiatry Cohort investigators and the MVP Core Acknowledgement are provided in the Supplemental

Materials. We are grateful to Luke Evans for helpful discussion of the manuscript.

\section{References}

1. Whiteford, H. A. et al. Global burden of disease attributable to mental and substance use disorders: Findings from the Global Burden of Disease Study 2010. Lancet 382, 15751586 (2013).

2. Hunt, G. E., Large, M. M., Cleary, M., Lai, H. M. X. \& Saunders, J. B. Prevalence of comorbid substance use in schizophrenia spectrum disorders in community and clinical settings, 1990-2017: Systematic review and meta-analysis. Drug Alcohol Depend. 191, 234-258 (2018).

3. Castillo-Carniglia, A., Keyes, K. M., Hasin, D. S. \& Cerdá, M. Psychiatric comorbidities in alcohol use disorder. The Lancet Psychiatry 6, 1068-1080 (2019).

4. SAMHSA. 2018 National Survey on Drug Use and Health (NSDUH). (2019).

5. Brady, K. T., Killeen, T. \& Jarrell, P. Depression in alcoholic schizophrenic patients. Am. J. Psychiatry 150, 1255-1256 (1993).

6. Duke, P. J., Pantelis, C. \& Barnes, T. R. E. South Westminster Schizophrenia Survey. Alcohol use and its relationship to symptoms, tardive dyskinesia and illness onset. Br. J. Psychiatry 164, 630-636 (1994).

7. Drake, R. E. \& Wallach, M. A. Substance Abuse Among the Chronic Mentally III. Psychiatr. Serv. 40, 1041-1046 (1989).

8. PF, S., KS, K. \& MC, N. Schizophrenia as a complex trait: Evidence from a meta-analysis of twin studies. Arch. Gen. Psychiatry 60, 1187-1192 (2003).

9. Verhulst, B., Neale, M. C. \& Kendler, K. S. The heritability of alcohol use disorders: a meta-analysis of twin and adoption studies. Psychol. Med. 45, 1061-1072 (2015).

10. Kranzler, H. R. et al. Genome-wide association study of alcohol consumption and use disorder in 274,424 individuals from multiple populations. Nat. Commun. 10, 1499 (2019).

11. Reginsson, G. W. et al. Polygenic risk scores for schizophrenia and bipolar disorder associate with addiction. Addiction Biology (2017). doi:10.1111/adb.12496

12. Liu, M. et al. Association studies of up to 1.2 million individuals yield new insights into the 
genetic etiology of tobacco and alcohol use. Nat. Genet. 51, 237-244 (2019).

13. Sanchez-Roige, S. et al. Genome-Wide Association Study Meta-Analysis of the Alcohol Use Disorders Identification Test (AUDIT) in Two Population-Based Cohorts. Am. J. Psychiatry (2018). doi:https://doi.org/10.1176/appi.ajp.2018.18040369

14. Lam, M. et al. Pleiotropic Meta-Analysis of Cognition, Education, and Schizophrenia Differentiates Roles of Early Neurodevelopmental and Adult Synaptic Pathways. Am. J. Hum. Genet. 105, 334-350 (2019).

15. Lee, P. H. et al. Genomic Relationships, Novel Loci, and Pleiotropic Mechanisms across Eight Psychiatric Disorders. Cell 179, 1469-1482.e11 (2019).

16. Ruderfer, D. M. et al. Genomic Dissection of Bipolar Disorder and Schizophrenia, Including 28 Subphenotypes. Cell 173, 1705-1715.e16 (2018).

17. Zhou, H. et al. Genome-wide meta-analysis of problematic alcohol use in 435,563 individuals yields insights into biology and relationships with other traits. Nat. Neurosci. (2020). doi:10.1038/s41593-020-0643-5

18. Walters, R. K. et al. Transancestral GWAS of alcohol dependence reveals common genetic underpinnings with psychiatric disorders. Nat. Neurosci. 21, 1656-1669 (2018).

19. Willer, C. J., Li, Y. \& Abecasis, G. R. METAL: fast and efficient meta-analysis of genomewide association scans. Bioinformatics 26, 2190-2191 (2010).

20. Pardiñas, A. F. et al. Common schizophrenia alleles are enriched in mutation-intolerant genes and in regions under strong background selection. Nat. Genet. 50, 381 (2018).

21. Bigdeli, T. B. et al. Contributions of common genetic variants to risk of schizophrenia among individuals of African and Latino ancestry. Mol. Psychiatry 1-13 (2019).

22. Bhattacharjee, S. et al. A Subset-Based Approach Improves Power and Interpretation for the Combined Analysis of Genetic Association Studies of Heterogeneous Traits. Am. J. Hum. Genet. 90, 821-835 (2012).

23. Bulik-Sullivan, B. et al. An atlas of genetic correlations across human diseases and traits. Nat. Genet. 47, 1236 (2015).

24. Bulik-Sullivan, B. K. et al. LD Score regression distinguishes confounding from polygenicity in genome-wide association studies. Nat. Genet. 47, 291-295 (2015).

25. Watanabe, K., Taskesen, E., van Bochoven, A. \& Posthuma, D. Functional mapping and annotation of genetic associations with FUMA. Nat. Commun. 8, 1826 (2017).

26. de Leeuw, C. A., Mooij, J. M., Heskes, T. \& Posthuma, D. MAGMA: Generalized GeneSet Analysis of GWAS Data. PLoS Comput. Biol. 11, (2015).

27. Liberzon, A. et al. Molecular signatures database (MSigDB) 3.0. Bioinformatics 27, 17391740 (2011).

28. Aguet, F. et al. Genetic effects on gene expression across human tissues. Nature 550, 204-213 (2017).

29. Zhu, Z. et al. Integration of summary data from GWAS and eQTL studies predicts complex trait gene targets. Nat. Genet. 48, 481-487 (2016).

30. De Jager, P. L. et al. A multi-omic atlas of the human frontal cortex for aging and Alzheimer's disease research. Sci. Data 5, 180142 (2018).

31. Akbarian, S. et al. The psychencode project. Nat. Neurosci. 18, 1707 (2015).

32. Kapoor, M. et al. Analysis of whole genome-transcriptomic organization in brain to identify genes associated with alcoholism. Transl. Psychiatry 9, 89 (2019).

33. Zhu, Z. et al. Causal associations between risk factors and common diseases inferred from GWAS summary data. Nat. Commun. 9, 224 (2018).

34. Fromer, M. et al. Gene expression elucidates functional impact of polygenic risk for schizophrenia. Nature Neuroscience 19, 1442-1453 (2016).

35. Fisher, R. A. Statistical methods for research workers. Stat. methods Res. Work. (1934).

36. Finucane, H. K. et al. Heritability enrichment of specifically expressed genes identifies disease-relevant tissues and cell types. Nat. Genet. 50, 621-629 (2018). 
medRxiv preprint doi: https://doi.org/10.1101/2020.10.27.20220186; this version posted December 16, 2020. The copyright holder for this preprint (which was not certified by peer review) is the author/funder, who has granted medRxiv a license to display the preprint in perpetuity.

It is made available under a CC-BY-NC-ND 4.0 International license .

37. Lonsdale, J. et al. The Genotype-Tissue Expression (GTEx) project. Nat. Genet. 45, 580585 (2013).

38. Lu, Q. et al. A Powerful Approach to Estimating Annotation-Stratified Genetic Covariance via GWAS Summary Statistics. Am. J. Hum. Genet. 101, 939-964 (2017).

39. Lu, Q. et al. A statistical framework to predict functional non-coding regions in the human genome through integrated analysis of annotation data. Sci. Rep. 5, 10576 (2015).

40. Lu, Q. et al. Systematic tissue-specific functional annotation of the human genome highlights immune-related DNA elements for late-onset Alzheimer's disease. PLoS Genet. 13, e1006933 (2017).

41. Lu, Q., Powles, R. L., Wang, Q., He, B. J. \& Zhao, H. Integrative tissue-specific functional annotations in the human genome provide novel insights on many complex traits and improve signal prioritization in genome wide association studies. PLoS Genet. 12, e1005947 (2016).

42. Marees, A. T. et al. Potential influence of socioeconomic status on genetic correlations between alcohol consumption measures and mental health. Psychol. Med. 1-15 (2019).

43. Marees, A. T. et al. Genetic correlates of socio-economic status influence the pattern of shared heritability across mental health traits. medRxiv 2020.02.26.20028092 (2020). doi:10.1101/2020.02.26.20028092

44. Watanabe, K. et al. A global overview of pleiotropy and genetic architecture in complex traits. Nat. Genet. (2019). doi:10.1038/s41588-019-0481-0

45. Clarke, T.-K. et al. Genome-wide association study of alcohol consumption and genetic overlap with other health-related traits in UK Biobank $(\mathrm{N}=112,117)$. Molecular Psychiatry (2017). doi:10.1038/mp.2017.153 
medRxiv preprint doi: https://doi.org/10.1101/2020.10.27.20220186; this version posted December 16, 2020. The copyright holder for this preprint (which was not certified by peer review) is the author/funder, who has granted medRxiv a license to display the preprint in perpetuity.

It is made available under a CC-BY-NC-ND 4.0 International license .

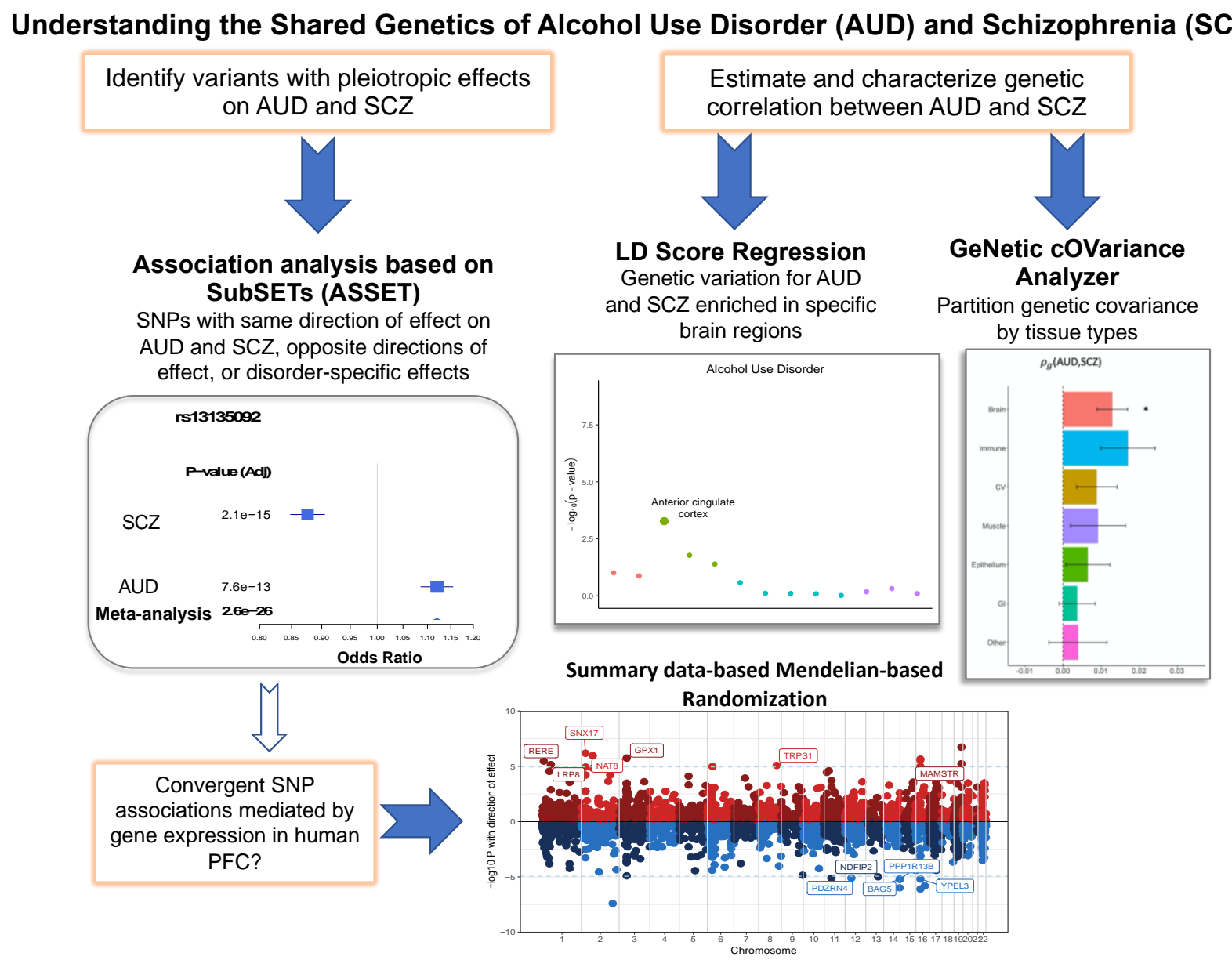

Figure 1. Conceptual overview of cross-disorder analysis of alcohol use disorder and schizophrenia. 


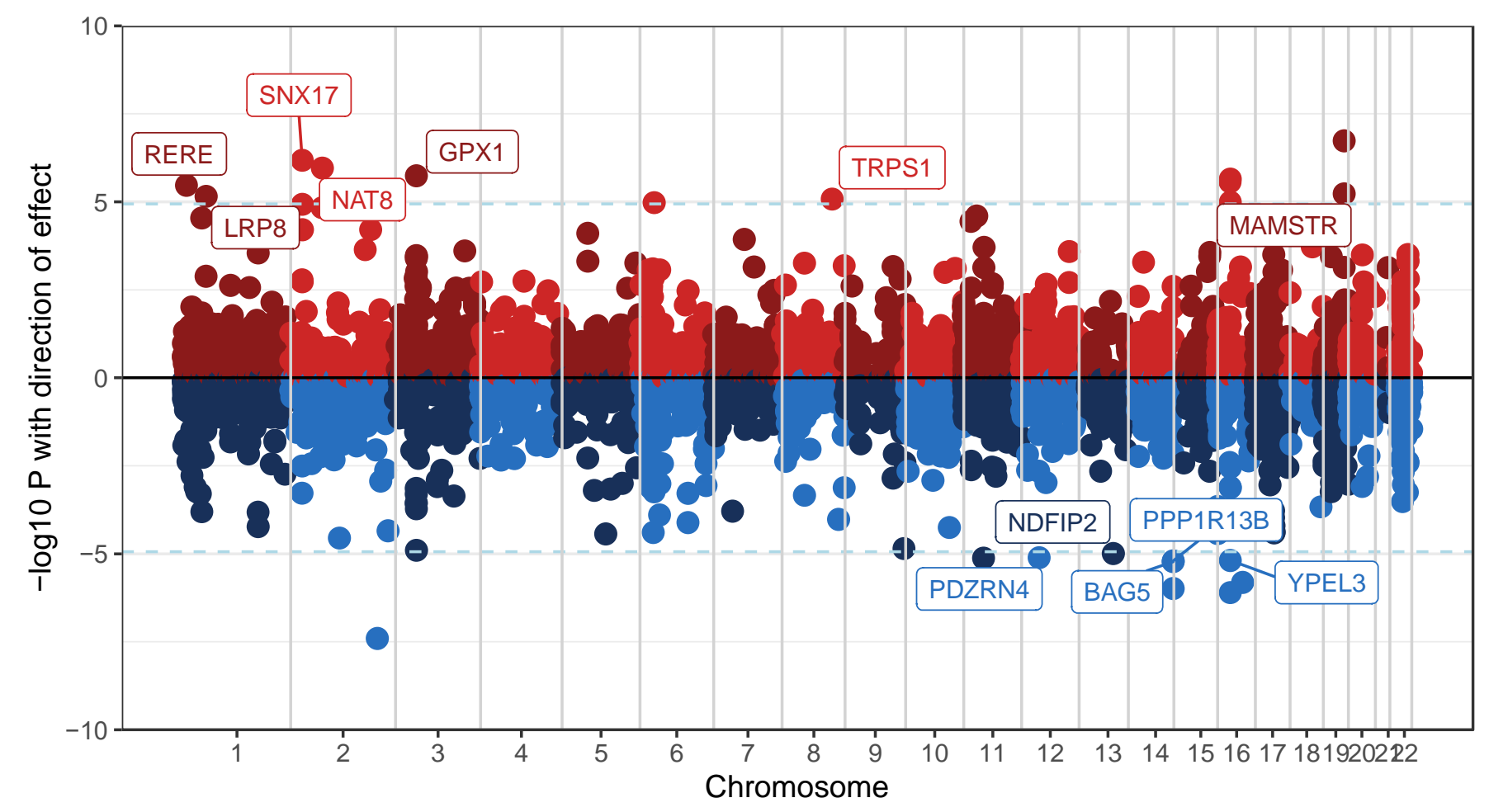

Figure 2. Genes in the convergent subset whose association with AUD and SCZ may be mediated by gene expression in the prefrontal cortex, analyzed using Summary data-based Mendelian Randomization (SMR). Genes in red show upregulated gene expression, and genes in blue show down-regulated gene expression. The 12 labeled genes are significant after Bonferroni corrections and were not excluded as pleiotropic outliers (HEIDI-outlier method $p>0.05$ ) 
medRxiv preprint doi: https://doi.org/10.1101/2020.10.27.20220186; this version posted December 16, 2020. The copyright holder for this preprint (which was not certified by peer review) is the author/funder, who has granted medRxiv a license to display the preprint in perpetuity.

It is made available under a CC-BY-NC-ND 4.0 International license .

\section{$\rho_{g}(\mathrm{AUD}, \mathrm{SCZ})$}

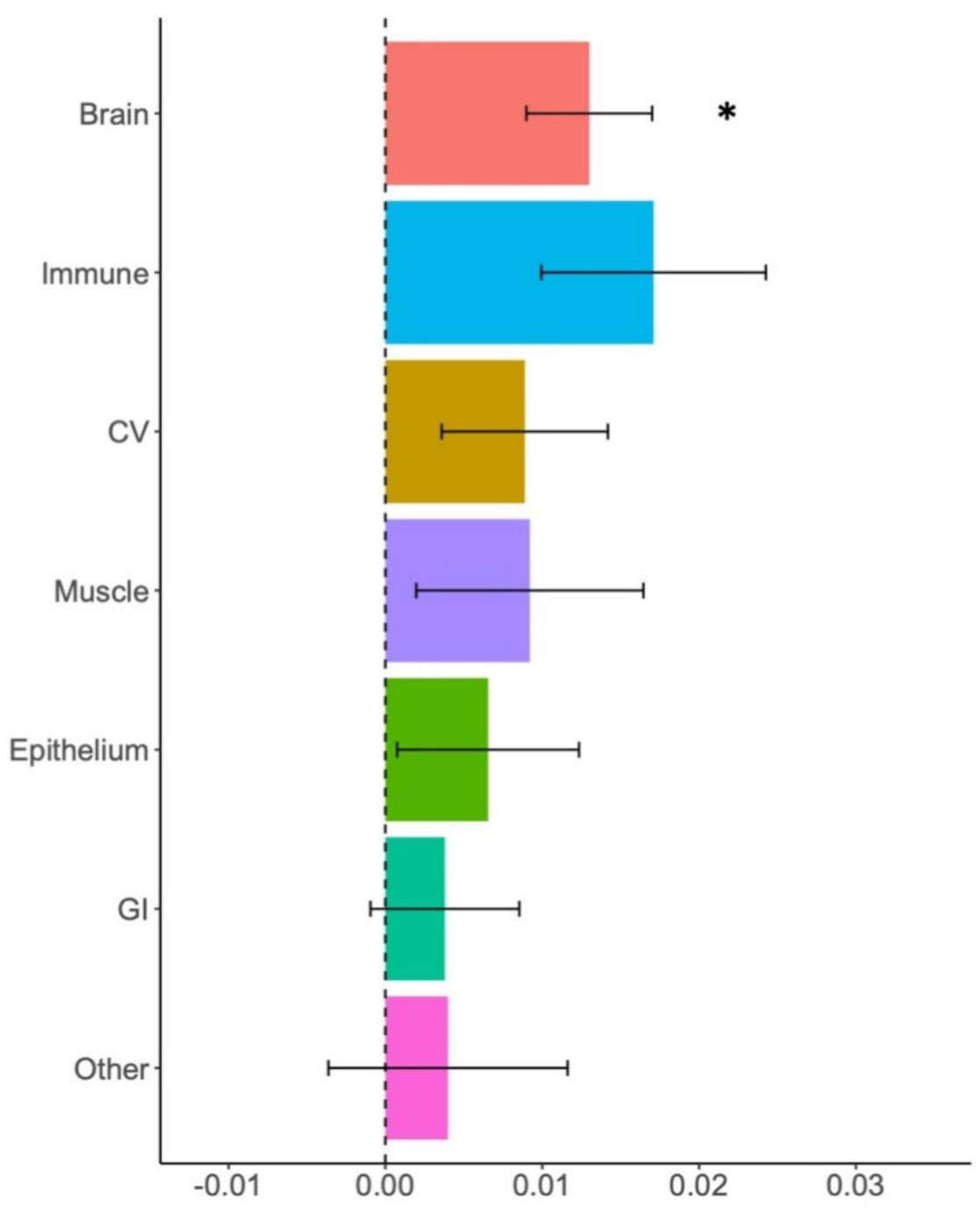

a. 
medRxiv preprint doi: https://doi.org/10.1101/2020.10.27.20220186; this version posted December 16, 2020. The copyright holder for this preprint (which was not certified by peer review) is the author/funder, who has granted medRxiv a license to display the preprint in perpetuity.

\section{$\rho_{g}(\mathrm{AUD}, \mathrm{SCZ})$}

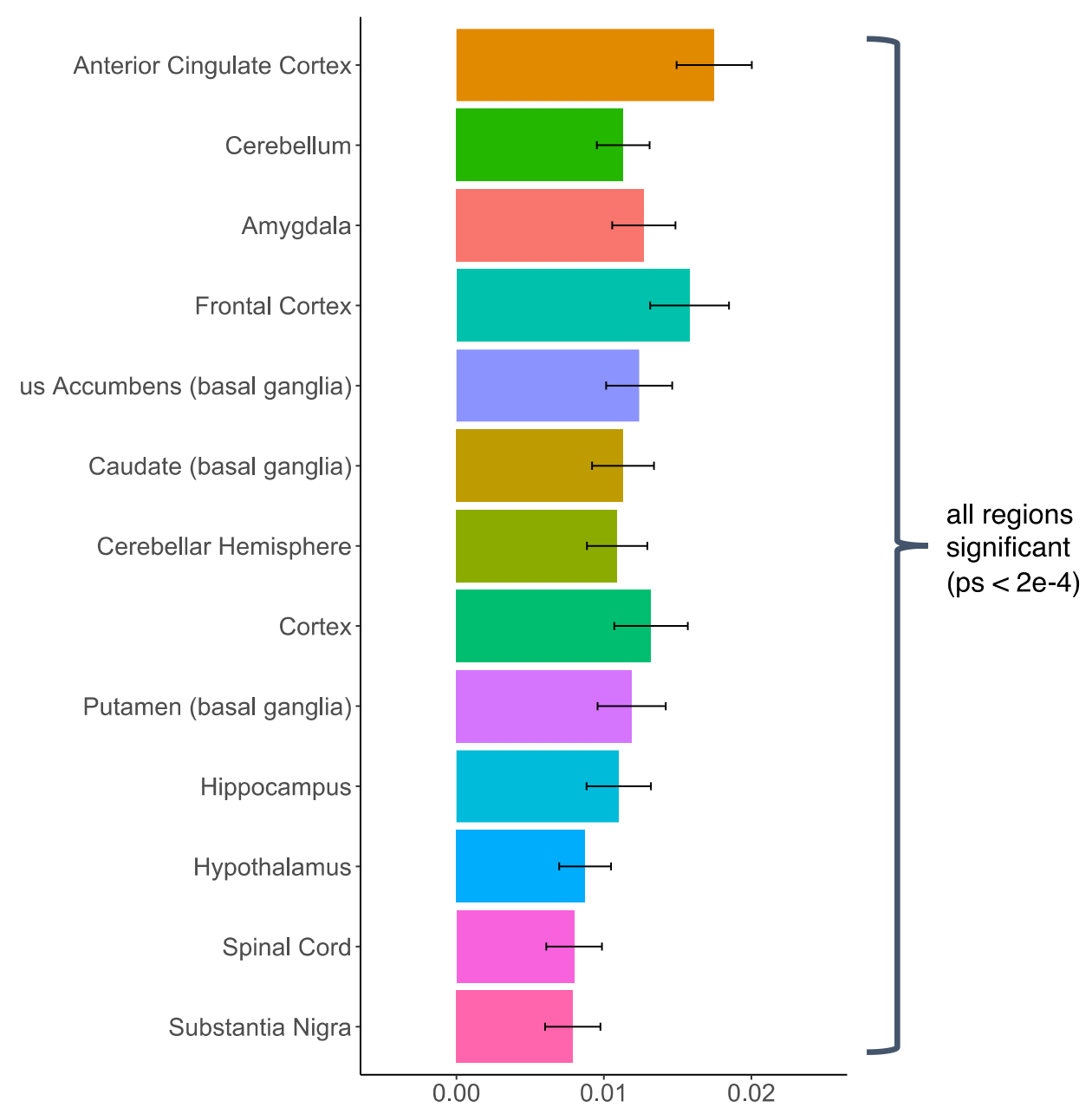

b.

Figure 3. Stratified genetic covariance between AUD and SCZ. a: stratified by broad tissue type. Tissue annotations were defined using the GenoSkyline-Plus annotations. Significant tissues are starred. $\mathrm{CV}=$ cardiovascular; $\mathrm{Gl}$ = gastrointestinal. b: stratified by 13 brain regions, defined using GTEx v6 gene expression data. 